\title{
Self-Consistent Scattering Calculation of Resonant Tunneling Diode Characteristics
}

\author{
J. P. SUN and G. I. HADDAD \\ Department of Electrical Engineering and Computer Science \\ The University of Michigan, Ann Arbor, MI 48109-2122
}

\begin{abstract}
We perform a self-consistent calculation of resonant tunneling diode (RTD) I-V characteristics including optical phonon scattering. The self-consistency is obtained by solving the Schrödinger equation and Poisson's equation iteratively with the Thomas-Fermi approximation used for the device contact regions. For evaluation of phonon-assisted current density, the optical phonon scattering in the quantum well is modeled using the optical model potential. Electron transverse momentum is also incorporated. The calculated current and electron wavefunction illustrate the optical model and effects of the phonon scattering on the current transport. The I-V characteristics we obtain from the model calculation are in good agreement with experimental results. This work manifests the importance of including self-consistency, optical phonon scattering, and electron transverse momentum in modeling realistic RTD structures.
\end{abstract}

\section{INTRODUCTION}

Although various models for the Resonant Tunneling Diodes (RTDs) have been developed, research for an accurate model of RTD suitable for device routine analysis is ongoing as the results of modeling calculations are generally not very satisfactory for a critical comparison between calculated and experimental data with discrepancies typically found in the valley current, and in the peak and valley voltages. Within the effective mass - envelope function framework, several formulations incorporating optical phonon scattering processes have been proposed for simulation of RTD I-V characteristics with various degrees of success. In this report we present modeling results of RTD I-V characteristics when the self-consistency, optical phonon scattering, and electron transverse momentum are all taken into consideration.

\section{RTD MODEL}

The self-consistent scattering calculation in this work can be divided into three parts: (1) Thomas-Fermi calculation which solves the Fermi distribution and Poisson's equation iteratively and generates selfconsistent charge and potential distributions in the contact regions. (2) The quantum well calculation which solves the Schrödinger equation for the quantized charge distribution in the quantum well; the quantum charge is then iterated with Poisson's equation to obtain self-consistent charge and potential distributions over the entire device. (3) Solution of scattering states for the Schrödinger equation whose potential term consists of both the self-consistent device potential and the imaginary optical potential introduced for inelastic phonon scattering process [1, 2]. To evaluate the phonon-assisted current density, 
for a specified bias voltage and temperature, eigenstate electrons with the Fermi distribution are incident upon the double-barrier structure, scattered elastically by the conduction band profile and inelastically in the quantum well by the optical potential, $-i \mathrm{~W}_{0}$. The device structural and doping specifications are incorporated in all the three parts. Details for the self-consistent solution and electron transverse momentum have been described elsewhere $[3,4,5]$. In steady state, the electron current density is attenuated by the optical potential at a rate of $2 W_{0} /$, corresponding to a scattering time $\tau=/ 2 W_{0}$. The probability of finding electrons in the quantum well is reduced corresponding to an electron mean free path given by $\lambda=v \tau$, where $v=\sqrt{ } 2 E / m *$ is the electron velocity for an electron state and $E$ is the electron energy in the growth direction. A damping constant $\gamma$ for the wavefunction in the quantum well can then be defined accordingly, $\gamma=1 /(2 v \tau)=\sqrt{ } m * / 2 E / \tau$. This allows us to express the electron wavefunction in the well as a linear combination of damping waves $\exp [i(k+i \gamma) x]$ and $\exp [-i(k+i \gamma) x]$. The one-dimensional, time-independent Schrödinger equation now reads,

$$
\begin{aligned}
& \frac{-\hbar^{2}}{2} \frac{\partial}{\partial x}\left[\frac{1}{m^{*}(x)} \frac{\partial \psi(x)}{\partial x}\right]- \\
& \quad\left[E+\frac{\hbar^{2} k_{\|}^{2}}{2 m^{*}(x)}-V(x)-i W_{0}(E, x)\right] \psi(x)=0
\end{aligned}
$$

where $m^{*}$ is the variable electron effective mass, $k_{\|}$is electron transverse momentum, and $\tau$ the value of the scattering time of hot electrons in the quantum well.

$$
\begin{aligned}
\frac{1}{\tau}=S_{o p}\left[1+\frac{2}{\exp \left(\hbar \omega_{o p} / k_{B} T\right)-1}\right], \\
E-E_{0} \geq \hbar \omega_{o p}(\text { emission/absorption) } \\
\frac{1}{\tau}=S_{o p}\left[\frac{1}{\exp \left(\hbar \omega_{o p} / k_{B} T\right)-1}\right], \\
\left|E-E_{0}\right|<\hbar \omega_{o p} \quad \text { (absorption) }
\end{aligned}
$$

where $S_{o p}$ can be calculated for the specific material, $\hbar \omega_{o p}$ the energy of optical phonons, $E_{0}$ the resonant energy, $k_{B}$ the Boltzmann constant, and $T$ is the absolute temperature. The inelastic scattering potential is then related to energy, material and temperature. The total transmission $T_{t o t}$ is the sum of coherent transmission $T_{c o h}$ and incoherent transmission $T_{\text {incoh }}$, and an attenuation coefficient is defined as $A=1-T_{c o h}-R_{c o h}$, where $R_{c o h}$ is the coherent reflection coefficient. After a single collision event, the attenuated part is assumed to tunnel in both $-x$ and $+x$ directions in proportion to the transmission coefficient for each barrier, denoted respectively as $T_{R}$ for the barrier to the right hand side and $T_{L}$ for the barrier to the left hand side of the quantum well. 5 The transmission from region $\mu$ to region $v$ is calculated to be the squared ratio of the transmitted electron flux to the incident flux,

$$
T_{\mu v}=\frac{\left|C_{v}\right|^{2}}{\left|A_{\mu}\right|^{2}} \frac{k_{v}}{k_{\mu}} \frac{m_{\mu}^{*}}{m_{v}^{*}}
$$

where $C_{v}$ is transmitted wave amplitude, with associated effective mass $m_{v}^{*}$ and wavevector $k_{v}$, while $A_{\mu}$ is incident wave amplitude, with $m_{\mu}^{*}$ and $k_{\mu}$ in the respective regions. The wave amplitudes are available from the numerical solution of Eq. (1) for a particular self-consistent potential $V(x)$. The total transmission through the double-barrier structure is then

$$
T_{\text {tot }}=T_{c o h}+T_{\text {incoh }}=T_{c o h}+\frac{A T_{R}}{T_{L}+T_{R}}
$$

The current transport components described as such are depicted in Fig. 1, the current attenuation and
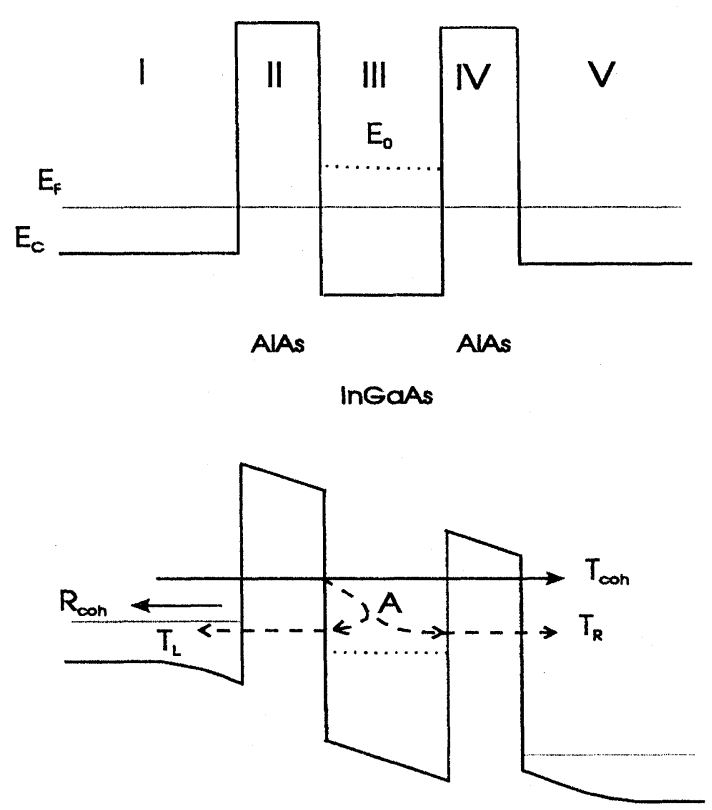

FIGURE 1 Current transport components in an RTD structure 
Phonon Scattering in RTD

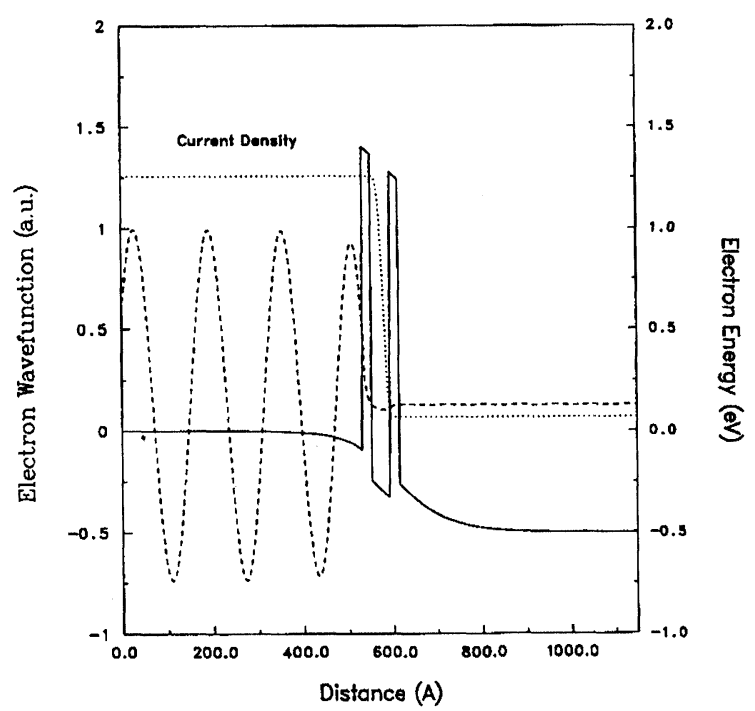

FIGURE 2 Calculated current density and electron wavefunction the RTD at bias voltage of $0.5 \mathrm{~V}$. Self-consistent case

electron wavefunction as calculated for an electron state due to the phonon scattering are plotted in Fig. 2, which illustrate the optical model. The integration of the total transmission over the energy then yields the device current.

\section{RESULTS}

Numerical simulations are performed for an RTD

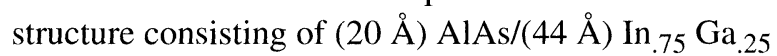
As/(20 ̊) AlAs double-barrier quantum well with a contact doping of $1.0 \times 10^{18} \mathrm{~cm}^{-3}$. The barrier height is taken to be $1.5 \mathrm{eV}$ between the contact and barrier and $1.61 \mathrm{eV}$ between the barrier and quantum well, with the electron effective mass being $0.044 m_{0}$, $0.092 m_{0}$, and $0.034 m_{0}$ in the contact, barrier, and well regions, respectively. Electron transverse momentum of $3.2 \times 10^{6} \mathrm{~cm}^{-1}$ is used. The optical phonon energy, $\hbar \omega_{o p}$, is estimated to be $32 \mathrm{meV}$ and $S_{o p}=1.25 \times 10^{13}$ is calculated using formulas similar to that in [6]. The calculated J-V characteristics of the RTD, at $77 \mathrm{~K}$ and $300 \mathrm{~K}$, for cases with and without the phonon scattering, are plotted in Fig. 3 with the

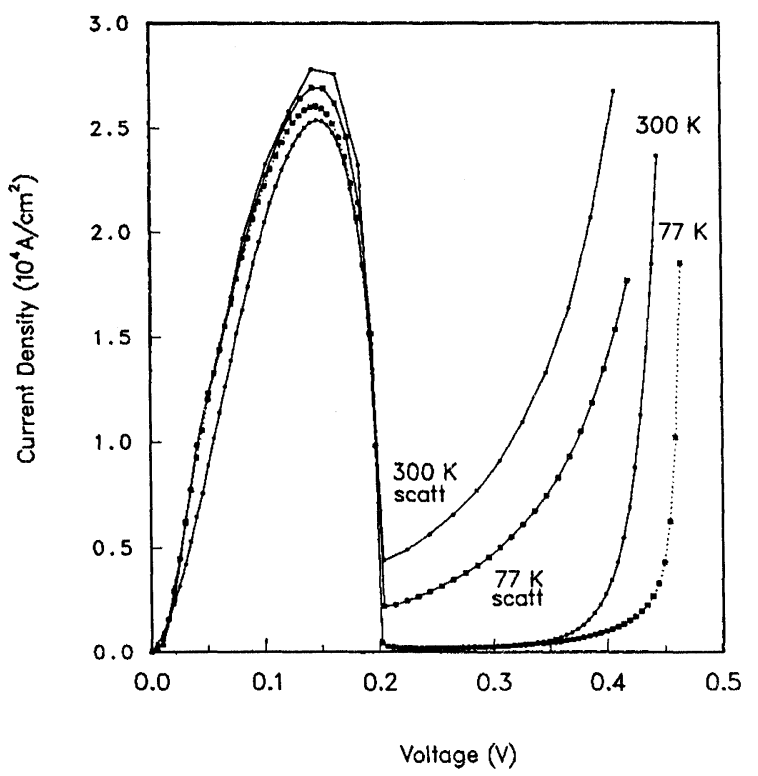

FIGURE 3 Calculated RTD J-V characteristics with and without phonon scattering. Non-self-consistent case

peak current density and peak-to-valley ratio very close to the experimental values at these temperatures and that the valley current density increases with temperature as expected. Note that for the results in Fig. 3, self-consistency and electron transverse momentum were not included. To obtain a good agreement between calculated and experimental peak and valley voltages, as shown in Fig. 4, the self-consistency and electron transverse momentum are incorporated to take into account effects of space charge and effective barrier lowering [5,7].

\section{CONCLUSION}

For realistic RTD I-V characteristics, inelastic phonon scattering, self-consistency and electron transverse momentum have been incorporated in the present model to give good agreement between calculated and experimental results. The calculated electron current and wavefunction profiles illustrate the effect of the phonon scattering in the quantum well. This approach is conceptually simple and computationally efficient. On the other hand, as a number of approxi- 


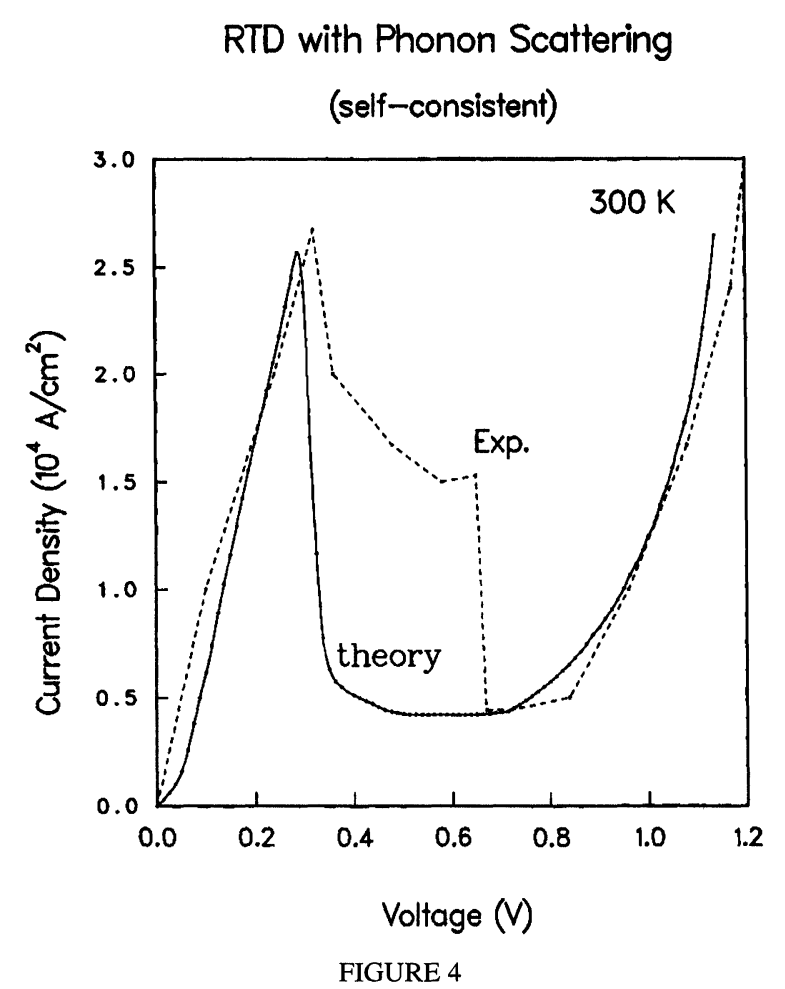

mations is involved, systematic tests on variations of device material and structural parameters are needed to assess the applicability of the present model. For more microscopic details of RTD device physics, one may have to invoke models based on quantum kinetic formalisms or many-body theories.

\section{Acknowledgements}

The authors wish to thank Dr. R. K. Mains, Dr. J. N. Schulman of Hughes Research Labs, and Dr. Y. Zohta of Tokyo Engineering University, for their very helpful discussions, and Dr. W. L. Chen for providing the RTD's experimental curve. This work was supported by the ARO-URI program under contract No. DAAL03-92-G-0109.

\section{Refercnces}

[1] A. D. Stone and P. A. Lee, Phys. Rev. Lett., 54, 1196 (1985).

[2] Y. Zohta and H. Ezawa J. Appl. Phys., 72, 3584 (1992); Y. Zohta and T. Tanamoto, J. Appl. Phys., 74, 6996 (1993); J. Appl. Phys., 76, 2343 (1994).

[3] R. K. Mains, J. P. Sun and G. I. Haddad, Appl. Phys. Lett., 55, 371 (1989).

[4] J. P. Sun, R. K. Mains, W. L Chen, J. R. East and G. I. Haddad, J. Appl. Phys., 72, 2340 (1992).

[5] J. P. Sun, R. K. Mains, K. Yang and G. I. Haddad, J. Appl. Phys., 74, 5053 (1993).

[6] M. A. Littlejohn, T. H. Glisson, and J. R. Hauser, in GaInAsP Alloy Semiconductors, T. P. Pearsall (ed.), ch.10., p. 243, Wiley, 1982.

[7] J. N. Schulman and H. J. De Los Santos, to be presented at 1995 International Semiconductor Device Research Symposium, Charlottesville, Virginia. 

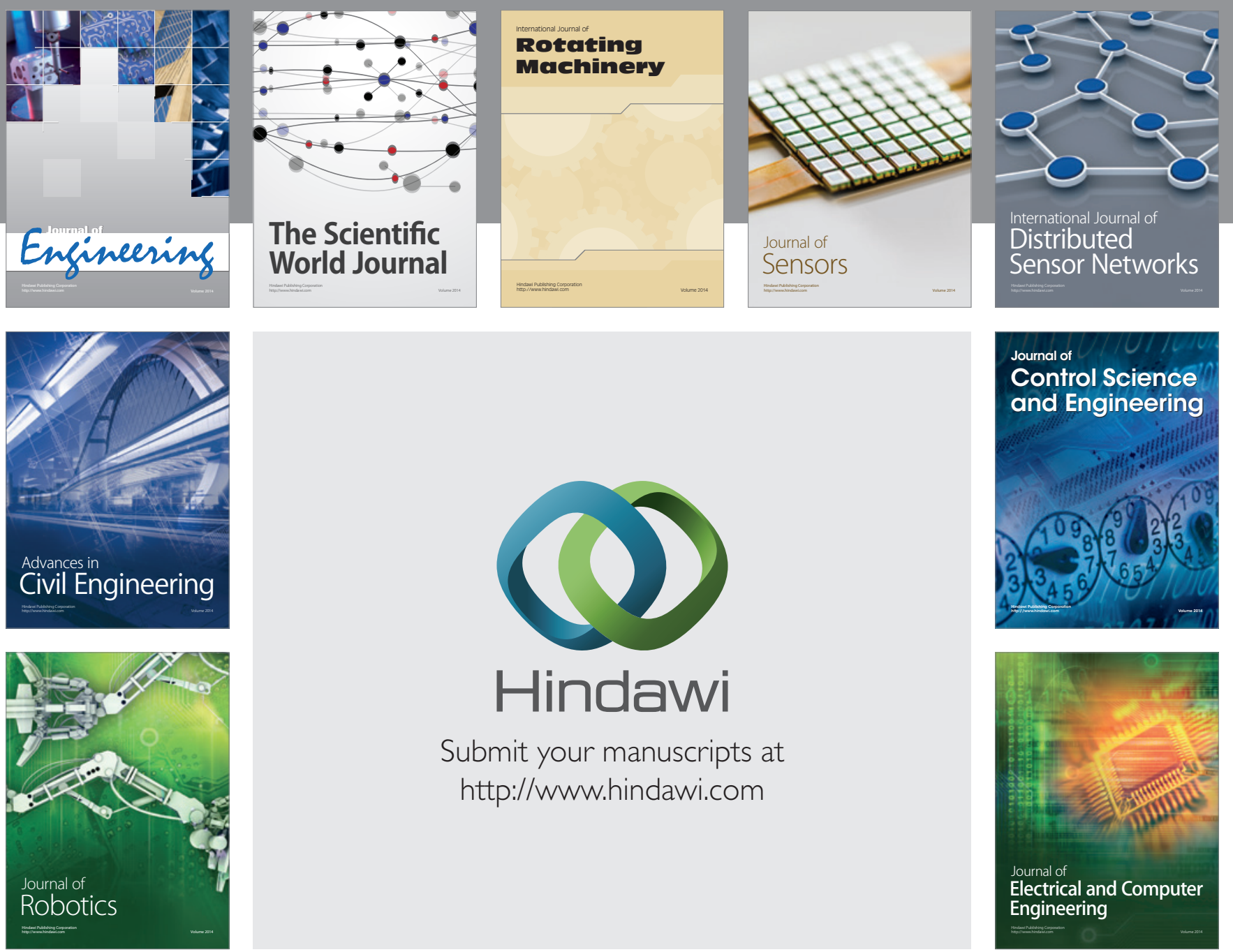

Submit your manuscripts at

http://www.hindawi.com
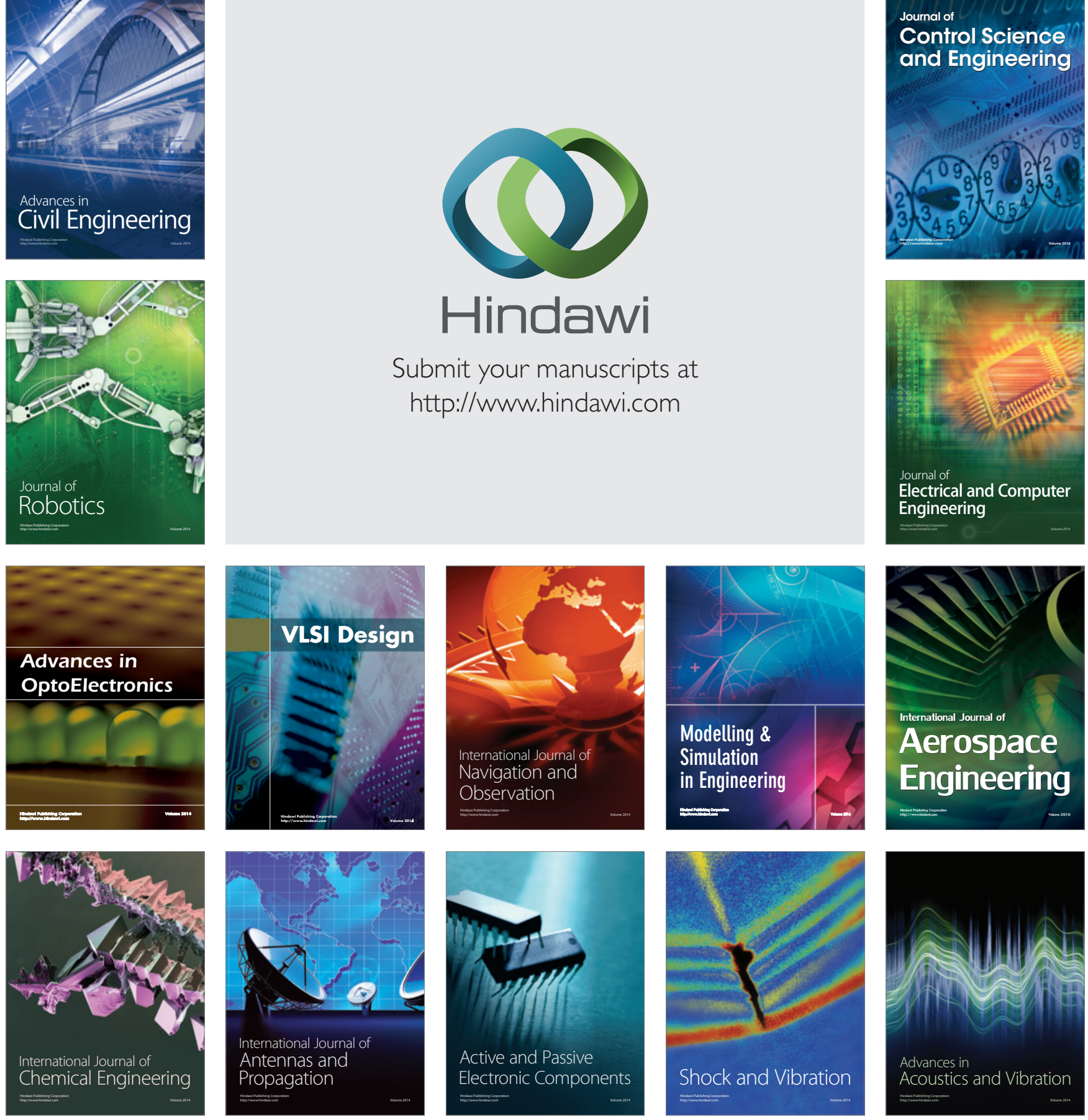